\title{
An Improved BER-Optimal Relay Selection Scheme for Decode-and- Forward Cooperative Networks
}

\author{
Yuhui Han, Mingji Yang and Aili Wang \\ School of Measurement and Control Technology and Communications Engineering \\ Higher Educational Key Laboratory for Measuring \& Control Technology and \\ Instrumentations of Heilongjiang \\ Harbin University of Science and Technology \\ Harbin, P.R.China \\ han_yuhui@126.com,yangmingji@126.com,aili925@hrbust.edu.cn
}

\begin{abstract}
We are concerned with the relay selection in a decode-and-forward cooperative network to minimize the bit error rate (BER) in a radio cell. This problem can be solved using maximum weighted $(M W)$ matching algorithm, greedy matching algorithm or worst-link-first (WLF) matching algorithm. Among the algorithms, WLF matching algorithm has achieved much attention for its being less complex while the achieved performance is high. In order to further reduce the computational complexity, we propose an improved WLF relay selection scheme, in which relays are selected according to instantaneous channel state information (CSI) and a cooperative threshold is set up to eliminate some unsatisfying candidate relays from the set of alternative candidate relays and thus reduce the amount of calculation. Theory analysis and simulation results both show that the improved WLF relay selection scheme proposed can be easily implemented and achieve almost the same BER performance as that of $W L F$ scheme while the computational complexity is much lower.
\end{abstract}

Keywords: cooperative communication; decode-and-forward; relay selection; worst-linkfirst; bit error rate

\section{Introduction}

Cooperative communication is a new spatial diversity technique, the basic idea of which is that a user and its partner/partners transmit cooperatively, thus providing space diversity ${ }^{[1,2]}$. Cooperative communication has been regarded as an effective method to combat fading in wireless channels and attracted great attention. Cooperative communication has good application prospects. It can be used to increase cell coverage, reduce energy consumption and improve the overall system performance. Cooperative communication has been considered for the next generation wireless communication systems.

Research shows that the relay selection scheme affects the performance of a cooperative network greatly. Optimal relay selection in cooperative networks is well studied ${ }^{[3-7]}$. The cooperative relay selection methods can be divided into distributed selection methods and centralized selection methods. Distributed selection methods are applicable to non-center networks, in which each node select relay/relays independently. Opportunistic relaying proposed in [3] and [4] is a typical distributed selection method. Centralized selection methods are applicable to networks with centers, in which the central node select relays for all nodes that need cooperation. Representative centralized selection methods include maximum weighted (MW) matching algorithm, greedy matching algorithm, worst-link-first (WLF) matching algorithm, and so on. Among them, WLF matching algorithm achieved great attention for its good performance and relatively low computational complexity ${ }^{[5]}$. 
In this paper, we consider single-relay decode-and-forward cooperative networks, and investigate the centralized relay selection scheme which takes the minimization of bit error rate (BER) as the optimization target. An improved WLF relay selection scheme is presented, in which a cooperative threshold is set up to eliminate some unsatisfying candidate relays from the set of alternative candidate relays and thus reduce the amount of calculation. Theory analysis and simulation results show that the proposed scheme can achieve almost the same BER performance as that of WLF scheme while the computational complexity is much lower.

The remainder of this paper is organized as follows. Section 2 describes the transmission model of single-relay decode-and-forward cooperative networks. In Section 3, we discuss the cooperative threshold for decode-and-forward cooperative networks. Based on the analysis of Section 3, an improved WLF relay selection scheme is proposed in Section 4. Section 5 gives the main conclusions of this paper.

\section{Transmission Model}

Single-relay decode-and-forward cooperative systems are considered in this paper. Figure 1 shows the transmission model. $h_{\mathrm{SD}}, h_{\mathrm{SR}}$ and $h_{\mathrm{RD}}$ are the channel fading coefficients of source to destination $(\mathrm{S}-\mathrm{D})$, source to relay $(\mathrm{S}-\mathrm{R})$ and relay to destination $(\mathrm{R}-\mathrm{D})$ links, respectively.

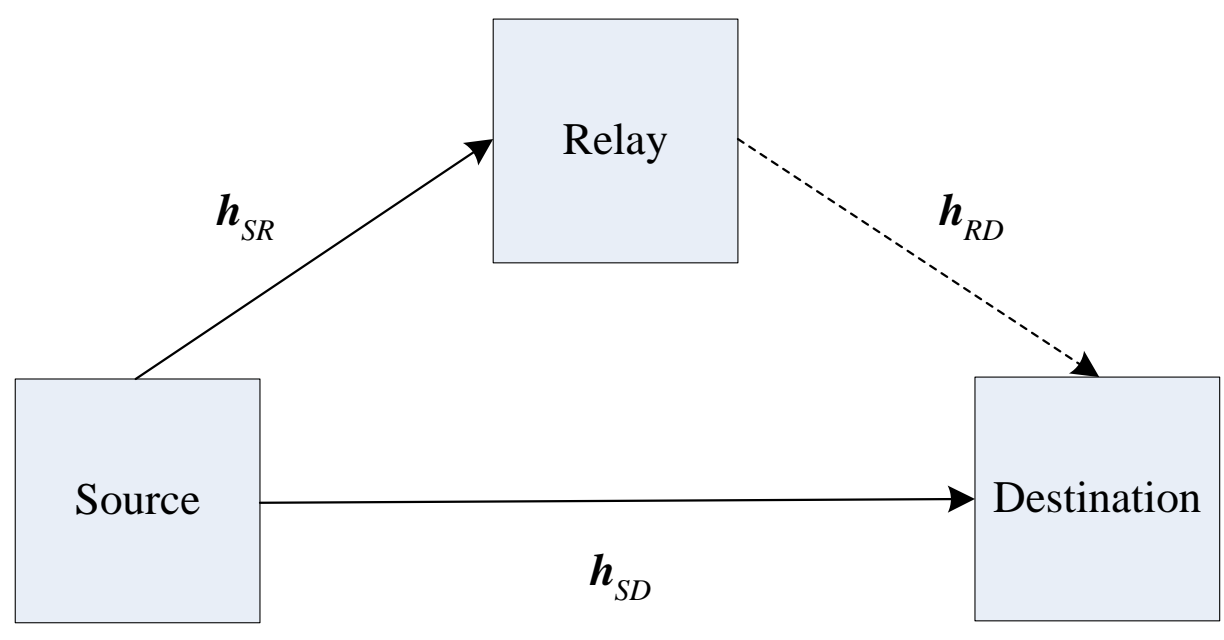

Figure 1. Transmission Model

The process of cooperative communication can be divided into two stages: in the first stage of cooperation (broadcasting stage), source transmits information to destination and relay, relay then decodes information; in the second stage of cooperation (relaying stage), relay forwards information to destination, destination combines signal transmitted from source in the first stage and signal transmitted from relay in the second stage according to maximum ratio combination method and then decodes information.

\section{Cooperative Threshold Analysis}

We consider quasi-static and flat fading channel. It is supposed that the transmit power of each node is 1 and binary phase shift keying (BPSK) modulation is adopted.

We can write the instantaneous BER of direct transmission as

$$
p_{e_{-} D}=Q\left(\sqrt{2 \gamma_{S D}}\right)
$$


where $Q(\cdot)$ is defined as

$$
Q(x)=\frac{1}{\sqrt{2 \pi}} \int_{x}^{\infty} e^{-\frac{t^{2}}{2}} d t
$$

$\gamma_{S D}$ is the instantaneous signal to noise ratio (SNR) of $\mathrm{S}-\mathrm{D}$ link,

$$
\gamma_{S D}=\frac{\left|h_{S D}\right|^{2}}{N_{0}},
$$

$N_{0}$ is the average power of additive white Gaussian noise (AWGN).

The instantaneous BER of decode-and-forward cooperation can be written as

$$
p_{e_{-} D F}=p_{e 1} \times \alpha+\left(1-p_{e 1}\right) \times p_{e 2}
$$

$p_{e 1}$ denotes the instantaneous BER at relay in the first stage of cooperation,

$$
p_{e 1}=Q\left(\sqrt{2 \gamma_{S R}}\right)
$$

where $\gamma_{S R}$ is the instantaneous SNR of S-R link.

$p_{e 2}$ denotes the instantaneous BER at destination in the second stage of cooperation, on the condition that relay correctly decode the information received from source in the first stage of cooperation,

$$
p_{e 2}=Q\left(\sqrt{2\left(\gamma_{S D}+\gamma_{R D}\right)}\right)
$$

where $\gamma_{R D}$ is the instantaneous SNR of $\mathrm{R}-\mathrm{D}$ link.

$\alpha$ is used to denote the instantaneous BER at destination in phase 2, on the condition that relay incorrectly decode the information received from source in the first stage of cooperation.

If $\gamma_{S D} \geq \gamma_{R D}$, we have

$$
\alpha=Q\left(\sqrt{\frac{2\left(\gamma_{S D}-\gamma_{R D}\right)^{2}}{\gamma_{S D}+\gamma_{R D}}}\right)
$$

If $\gamma_{S D}<\gamma_{R D}, \alpha$ can be calculated as

$$
\alpha=1-Q\left(\sqrt{\frac{2\left(\gamma_{S D}-\gamma_{R D}\right)^{2}}{\gamma_{S D}+\gamma_{R D}}}\right)
$$

If the SNR of S-D link is high, cooperation is usually not needed. So we only consider the scenario that the SNR of S-D link is low. Now we analyze two ideal cases. 


\subsection{High SNR in R - D link and low SNR in S - D link}

We can see from (4) to (8) that if the SNR of R-D link is very high and the SNR of S D link is low, $p_{e 2}$ will approximately equal to 0 and $\alpha$ will approximately equal to 1 . In this case, we have

$$
p_{e_{-} D F} \approx p_{e 1}=Q\left(\sqrt{2 \gamma_{S R}}\right)
$$

Compare (9) to (1), it can be seen that if the SNR of R-D link is very high and the SNR of $\mathrm{S}-\mathrm{D}$ link is low, when $\gamma_{S R}>\gamma_{S D}$, the instantaneous BER of DF cooperation will be lower than that of direct transmission; when $\gamma_{S R}<\gamma_{S D}$, the instantaneous BER of DF cooperation will be higher than that of direct transmission.

\subsection{High SNR in S - R link and low SNR in S - D link}

If the SNR of $S-R$ link is very high and the SNR of $S-D$ link is low, it can be seen from (5) that $p_{e 1}$ will approximately equal to 0 . In this case, we get

$$
p_{e_{-} D F} \approx p_{e 2}=Q\left(\sqrt{2\left(\gamma_{S D}+\gamma_{R D}\right)}\right)
$$

Compare (10) with (1), we can see that when the SNR of S-R link is very high and the SNR of $\mathrm{S}-\mathrm{D}$ link is low, because $\gamma_{S D}+\gamma_{R D}>\gamma_{S D}$, the instantaneous BER of DF cooperation will be lower than that of direct transmission.

According to the analysis above, it can be concluded that if we want a notable improvement of BER performance, the following threshold for cooperation should be satisfied,

$$
\gamma_{S R}>\gamma_{S D}
$$

If this cooperative threshold is not satisfied, the BER performance of DF cooperation cannot be much better than that of direct transmission; it may be close to or even poor than the BER performance of direct transmission. When this cooperative threshold is satisfied, the DF cooperation may have better BER performance than direct transmission.

\section{Improved WLF Relay Selection Scheme}

Various relay selection schemes have been proposed, such as greedy matching algorithm, MW matching algorithm, WLF matching algorithm, and so on. Among them, WLF matching algorithm has attracted much interest. Its basic idea is that the user with the worse channel condition is prior to other users in choosing its partner. WLF matching algorithm can achieve high performance with low complexity.

In this paper, we consider the scenario that the moving speeds of mobile stations are low, thus the channel state can be looked as constant in an enough long period. In this scenario, we can select relays for users according to instantaneous channel state information (CSI). We assume that the base station (BS) has perfect CSI of mobile station (MS) to BS links and MS to MS links. Our optimization goal is to minimize the BER of the cooperative communication system.

Based on the analysis in Section 3, we propose an improved WLF relay selection scheme. A special threshold, as shown in (11), is set up to avoid unnecessary cooperation and narrow the alternative relay field. Here, a non-reciprocal scenario is considered. 
Figure 2 is the flow chart of the improved WLF scheme proposed. Here, $\mathbf{U}_{\mathbf{u n}}$ denotes the set of users that haven't find their relays, $\mathbf{R}_{\mathbf{u n}}$ denotes the set of users that haven't been selected as other users' relays.

The improved WLF scheme proposed can be expressed as follows.

1) The BS selects a user $i$ from $\mathbf{U}_{\mathbf{u n}}$ with the smallest $\gamma_{S D}$.

2) The BS selects a user $j$ from $\mathbf{R}_{\mathbf{u n}}$ as the relay of user $i$ such that the theoretical instantaneous BER calculated according to (4) to (8) is the smallest one and satisfies the threshold as shown in (11). Then delete user $i$ from $\mathbf{U}_{\mathbf{u n}}$ and delete user $j$ from $\mathbf{R}_{\mathbf{u n}}$.

3) If there is no user $j$ satisfies the threshold as shown in (11) in $\mathbf{R}_{\mathbf{u n}}$, user $i$ will adopt direct transmission mode, and then delete user $i$ from $\mathbf{U}_{\mathbf{u n}}$.

4) Repeat 1), 2) and 3) until $\mathbf{U}_{\mathbf{u n}}$ is empty.

The simulation results are shown in Figure 3 and Figure 4. Here, we suppose that users are uniformly distributed in a circular area, BPSK modulation is employed, the channel is Rayleigh flat-fading and AWGN channel.

Figure 3 shows the average BERs of direct transmission and DF cooperative networks with different relay selection schemes: random selection scheme, WLF selection scheme and improved WLF selection scheme. From the figure, it can be seen that the BER performances of improved WLF scheme and WLF scheme are almost same and much better than the BER performance of direct transmission and random selection scheme. The BERs of WLF scheme and improved WLF scheme decrease while the number of users added. The reason is that more users means more alternative relays, and a user may find a better relay if the number of alternative relays increased.

Figure 4 shows the average calculated amount of relay selection for one user. Here, we assume that the calculated amount of calculating the theoretical instantaneous BER according to (4) to (8) once is 1 , and the calculated amount of comparing theoretical instantaneous BERs is neglected. It can be seen that the improved WLF selection scheme has a much lower average calculated amount than WLF selection scheme. The average calculated amount increased as the number of users added. This is because that with more alternative relays, more times of theoretical instantaneous BER calculation is needed. 


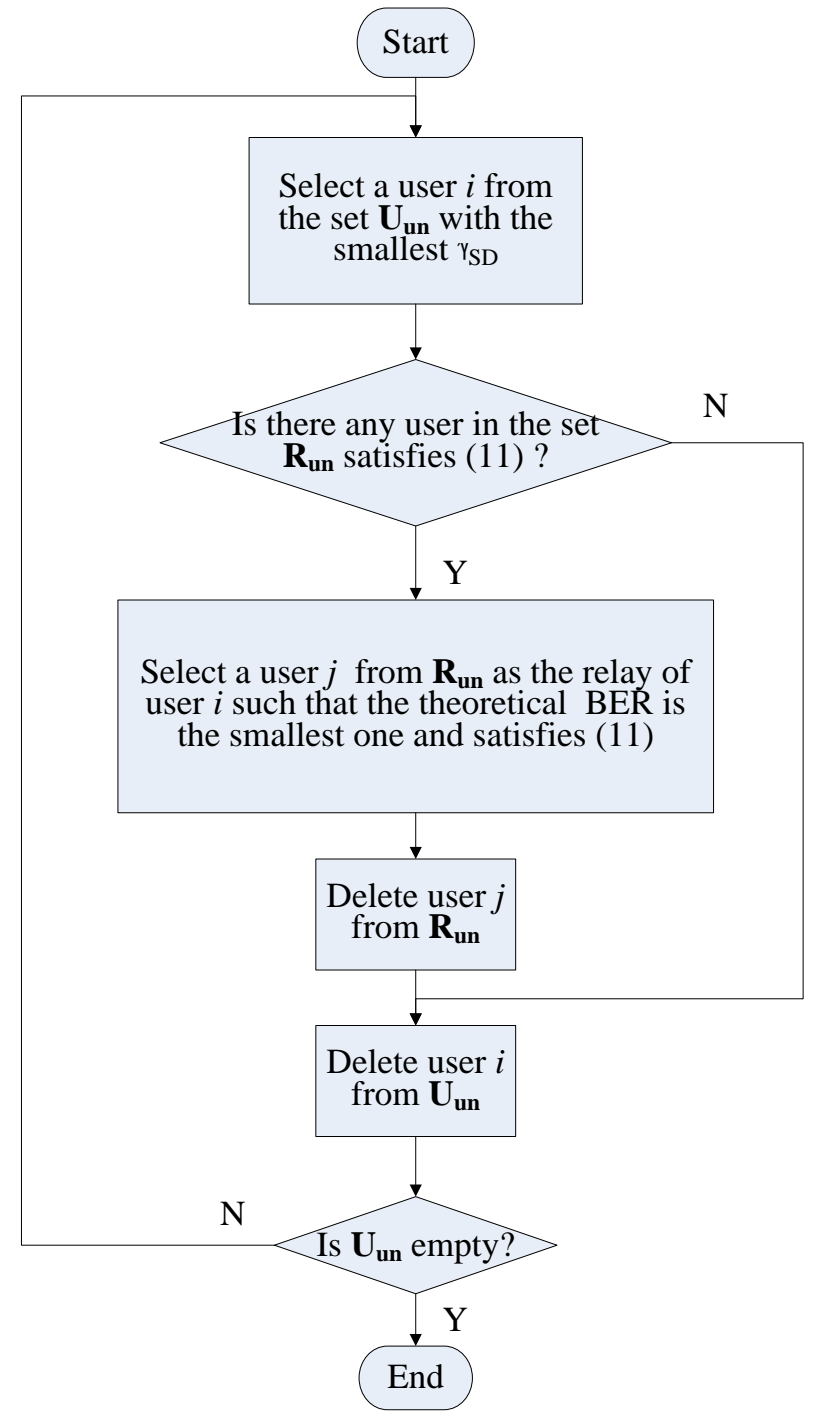

Figure 2. Flow Chart of the Improved WLF Scheme 


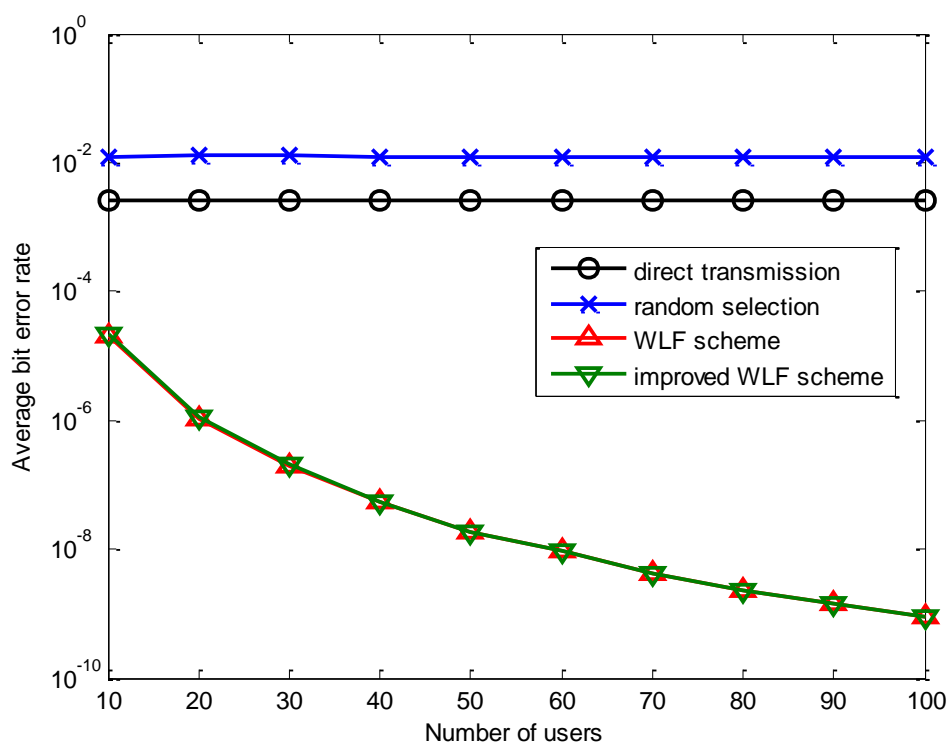

Figure 3. Average Bit Error Rate

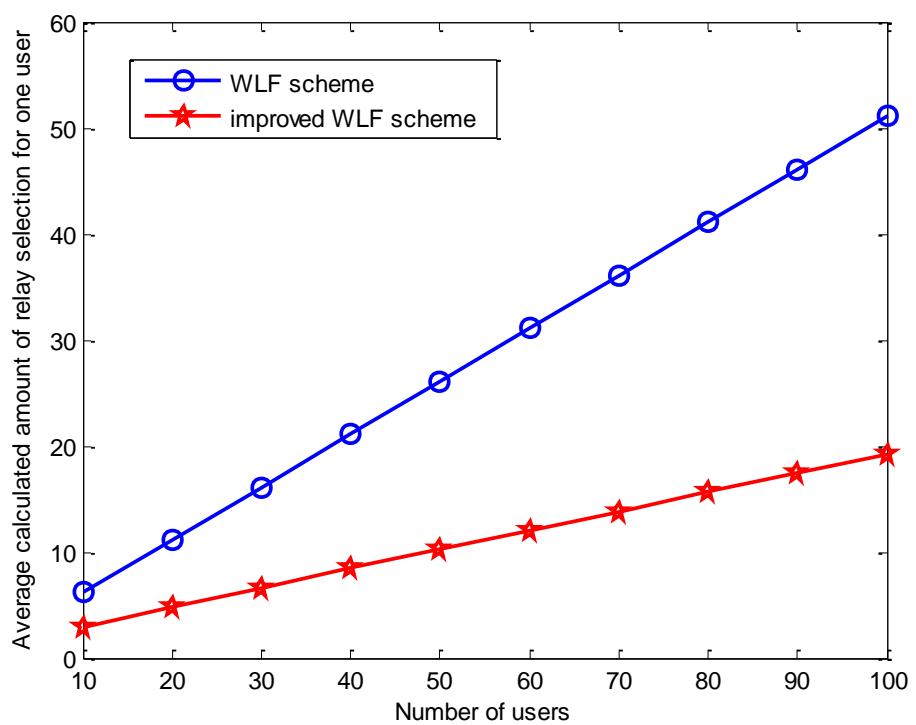

Figure 4. Average Calculated Amount

\section{Conclusions}

In this paper, we analyze the BER performance of single-relay DF cooperative networks. Then a cooperative threshold is proposed according to the analysis. Only when the threshold is satisfied, DF cooperation may have notable improvement of BER performance compared to direct transmission. An improved WLF relay selection scheme is proposed, which employ the cooperative threshold to reduce the number of alternative relays and thus decrease the computational complexity. Simulation results shows that the BER performance of the improved WLF relay selection scheme is almost the same as that of traditional WLF scheme, while it has a much lower computational complexity than WLF scheme. 


\section{Acknowledgements}

This work was supported by Heilongiiang Province Department of Education Science and Technology Research Project (12541143).

\section{References}

[1] A. Sendonaris, E. Erkip and B. Aazhang, "User Cooperation Diversity, Part I: System Description", IEEE Transaction on Communications, vol. 51, (2003), pp. 1927-1938.

[2] A. Sendonaris, E. Erkip and B. Aazhang, "User Cooperation Diversity", Part II: Implementation Aspects and Performance Analysis, IEEE Transaction on Communications, vol. 51, (2003), pp. 1939-1948.

[3] A. Bletsas, S. Hyundong, M. Z. Win and A. Lippman, "Cooperative Diversity with Opportunistic Relaying", Proceedings of the Wireless Communications and Networking Conference, (2006) April 3-6, Las Vegas, USA

[4] A. Bletsas, H. Shin and M. Z. Win, "Cooperative Communications with Outage-Optimal Opportunistic Relaying”, IEEE Transactions on Wireless Communications, vol. 6, (2007), pp. 3450-3460.

[5] V. Mahinthan, Lin Cai, J. W. Mark and X. Shen, "Maximizing Cooperative Diversity Energy Gain for Wireless Networks", IEEE Transactions on Wireless Communications, vol. 6, (2007), pp. 2530-2539.

[6] V. Mahinthan, Lin Cai, J. W. Mark and X. Shen, "Partner Selection Based on Optimal Power Allocation in Cooperative-Diversity Systems", IEEE Transactions on Vehicular Technology, vol. 57, (2008), pp. 511-520.

[7] Z. Lin, E. Erkip and A. Stefanov, "Cooperative Regions and Partner Choice in Coded Cooperative Systems", IEEE Transactions on Communications, vol. 54, (2006), pp. 1323-1334. 